\title{
НОРМОТЕНЗИВНАЯ ГЛАУКОМА ИЛИ АДЕНОМА ГИПОФИЗА?
}

*Эфендиева М.Э.

Нацииональный Центр Офтальмологии имени акад. Зарифы Алиевой, г. Баку, Азербайджан

$\mathbf{H}$ ормотензивная глаукома (НГ) традиционно определяется как прогрессирующая глаукоматозная оптическая нейропатия (ГОН) сопровождающаяся характерными изменениями диска зрительного нерва и поля зрения. Как правило, в постановке диагноза помогает присутствие факторов риска, таких как преклонный возраст, пол, наличие миопии, мигрени, периферический вазоспазм на фоне прогрессирующего снижения зрения, дефектов поля зрения и расширения глаукоматозной экскавации $[1,2,3]$.

Но, к сожалению НГ является одной из самых трудно диагностируемых нозологических форм открытоугольной глаукомы и как показал ретроспективный анализ литературы и клиническая практика, нередки случаи обнаружения аденомы гипофиза у пациентов с предполагаемой НГ [2,3,4,5,6,7,8,9,10,11]. По данным ряда авторов частота неверного диагностирования неглаукоматозной оптической нейропатии, развившейся вследствие компрессии аденомой гипофиза хиазмы и зрительного нерва составляет около 6,5-8\% $[6,11]$.

Согласно отчету "American Cancer Society" ежегодно в США диагностируется до 8000 опухолей гипофиза, при этом наиболее часто в $(80 \%)$ случаев встречается аденома гипофиза $[8,12]$. По статистическим данным в России и странах СНГ ежегодно выявляется около 3 тысяч вновь заболевших человек. С учетом многолетнего характера течения заболевания можно предположить, что число больных, требующих лечения или активного наблюдения, составляет порядка 80-100 тысяч человек, часть из которых становится инвалидами вследствие несвоевременно начатого или неадекватного лечения [13].

Аденома - доброкачественная опухоль, которая растет очень медленно, на протяжении ряда лет. По размерам ее разделяют на макроаденомы (более 10 мм) и микроаденомы (до *e-mail: metinefendi@mail.ru
10 мм), а клинически на гормонально активные и неактивные опухоли. Макроаденомы независимо от своей гормональной активности разрастаясь, могут вызывать компрессию близлежащих тканей, которая проявляется симптомами, характерными для объемного образования головного мозга: головными болями, нарушениями зрения, параличом глазодвигательных мышц, гидроцефалией и истечением спинномозговой жидкости из носа. В офтальмологической симптоматике раньше всего диагностируются изменения полей зрения при нормальной или слегка пониженной остроте зрения. Чаще встречаются различные формы битемпоральной гемианопсии (77\%), что объясняется тем, что крупная опухоль гипофиза при распространении кверху прорастает через диафрагму турецкого седла и сдавливает волокна в медиальной части хиазмы у нижней ее поверхности. Нередко отмечается более или менее значительное сужение носовых половин, что указывает на частичное поражение неперекрещенных волокон в хиазме. $[8,14,15]$. В постановке окончательного диагноза важную роль играет КТ или МРТ.

Цель. дифференциальная диагностика патологии зрения при макроаденоме и нормотензивной глаукоме.

Материал и методы. Под наблюдением находилась пациентка И., 59 лет, которая обратилась (13.12.12) с жалобами на снижение остроты зрения и выпадение поля зрения на левом глазу. Со слов пациентки она около 4-х лет отмечает снижение зрения на левом глазу, a 3 года как в поликлинике находится на диспансерном учете с диагнозом: нормотензивной глаукомы и получает антиглаукоматозное лечение (капли "простагландина" и "бетаблокатора"). В анамнезе гипертония, периодические головные боли. Объективный офтальмостатус: vis $\mathrm{OD}=0,8 /$ vis $\mathrm{OS}=0,08$ (экс.); уровень офтальтонуса OD/OS $=16 / 19$ мм.рт.ст. (под каплями). Придатки глаз и оп- 
тические среды были без изменений. На глазном дне: ДЗН OD - бледно- розовый, границы четкие, Э/Д=0,2-0,3, макуло-фовеолярный рефлексы выражены; ДЗН OS резко деколорирован в темпоральной половине, Э/Д=0,4-0,5, имеется незначительное смещение сосудистого пучка в носовую сторону, макуло-фовеолярный рефлексы слабо выражены. На обоих глазах отмечалось побледнение нейроретинального ободка (rim), а также сужение кали-

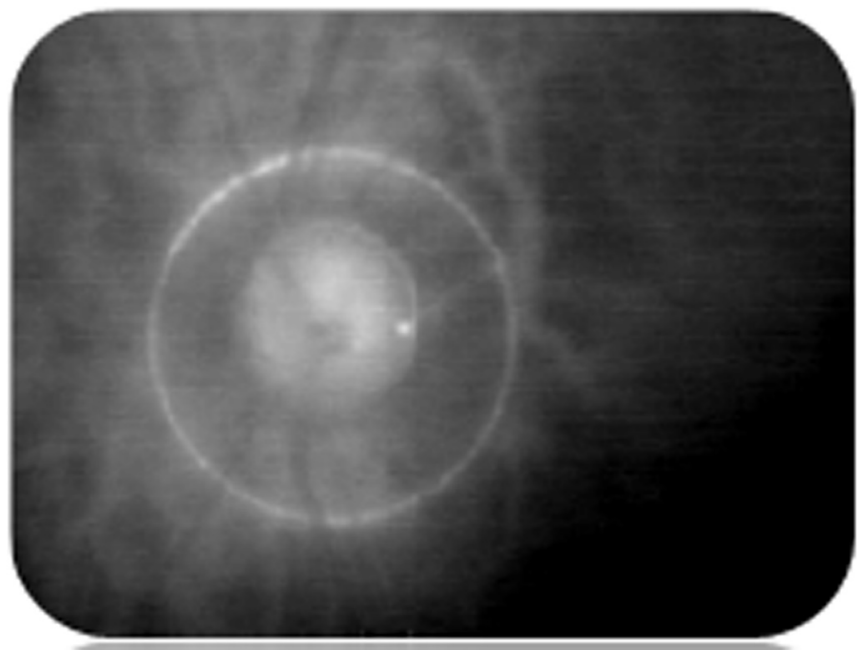

Левый глаз бра сосудов 1:3 (рис.1). При дополнительном обследовании выявлено: наличие относительного афферентного зрачкового дефекта (зрачок Маркуса-Гунна), а также отрицательный тест Амслера на левом глазу. Ультразвуковое допплерографическое исследование сосудов глаза показало резкое снижение скорости кровотока в центральной артерии сетчатки и задних цилиарных артериях на левом глазу, и незначительное на правом.

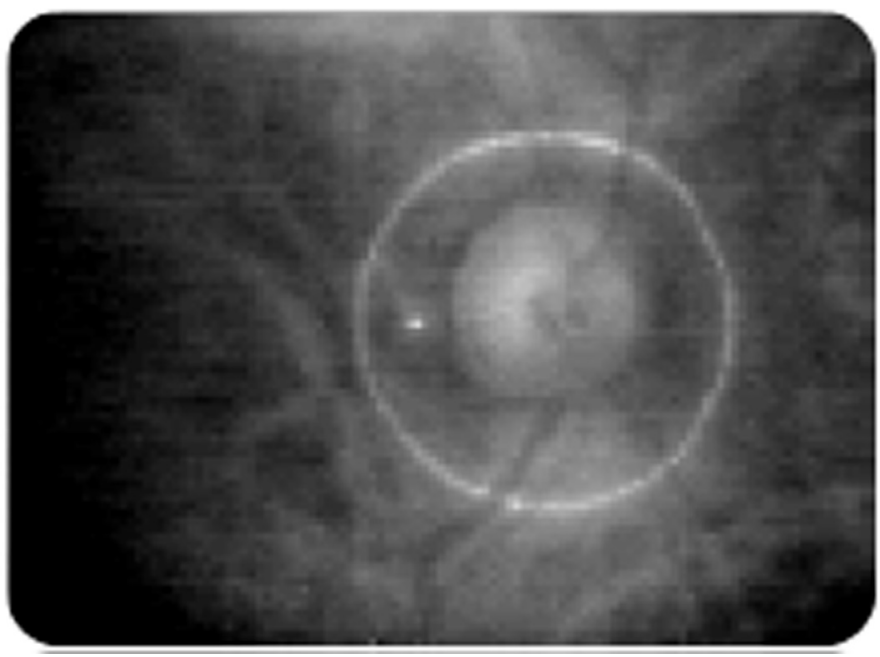

Правый глаз

Рис.1. Глазное дно

Учитывая ухудшение состояния пациентки, все дальнейшие обследования были перенесены на несколько дней позднее. Ей было рекомендовано отменить глазные капли. Спустя 4 дня (17.12.12): vis $\mathrm{OD}=0,8 /$ vis $\mathrm{OS}=0,1$ (экс.) уровень офтальтонуса OD/OS=19/17

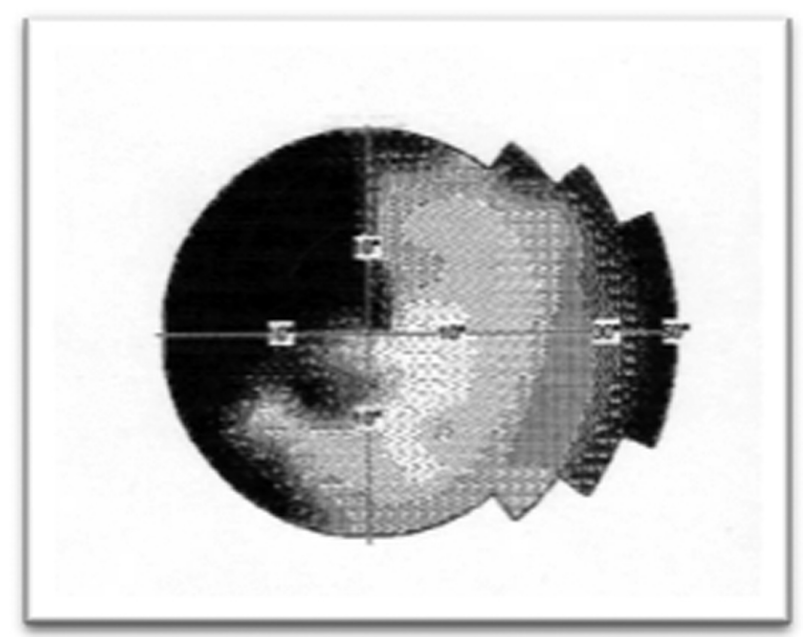

мм.рт.ст.(без капель). По данным компьютерной периметрии (TOMEY AP-2000 Automated Perimeter 91) выявлено сужение верхне- и нижне- височной границы до 150, а в верхнеи нижне-носовой границы до 300 на OD и на $\mathrm{OS}$ - сужение поля зрения в верхне-височном

Левый глаз

Рис.2. Поля зрения 

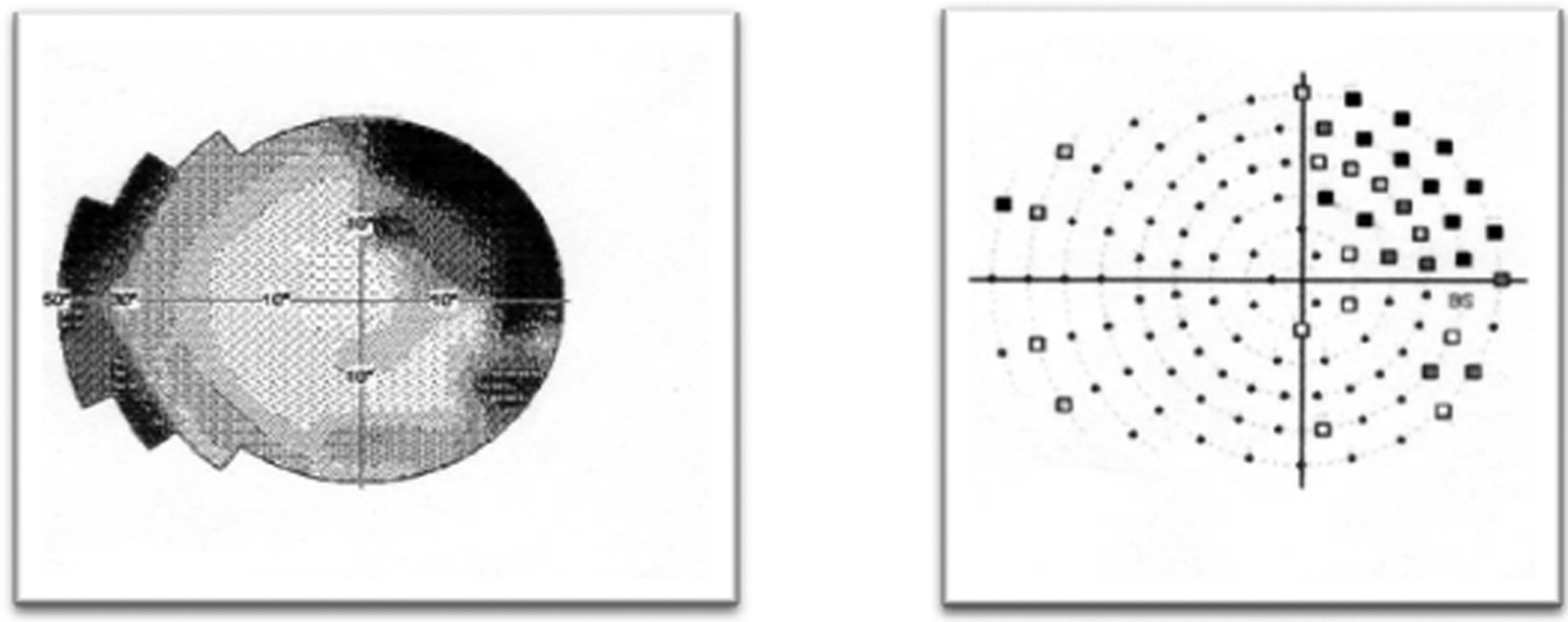

Правый глаз

Рис.2. Поля зрения

квадранте - до точки фиксации; нижне-височном квадрантах до 50, а в верхне- и нижне-носовом квадранте до 300 (Hemifield depression), что в целом можно расценивать как гипопсию височной половины поля зрения справа и темпоральную гемианопсию слева (рис. 2).

Согласно данным оптической когерентной

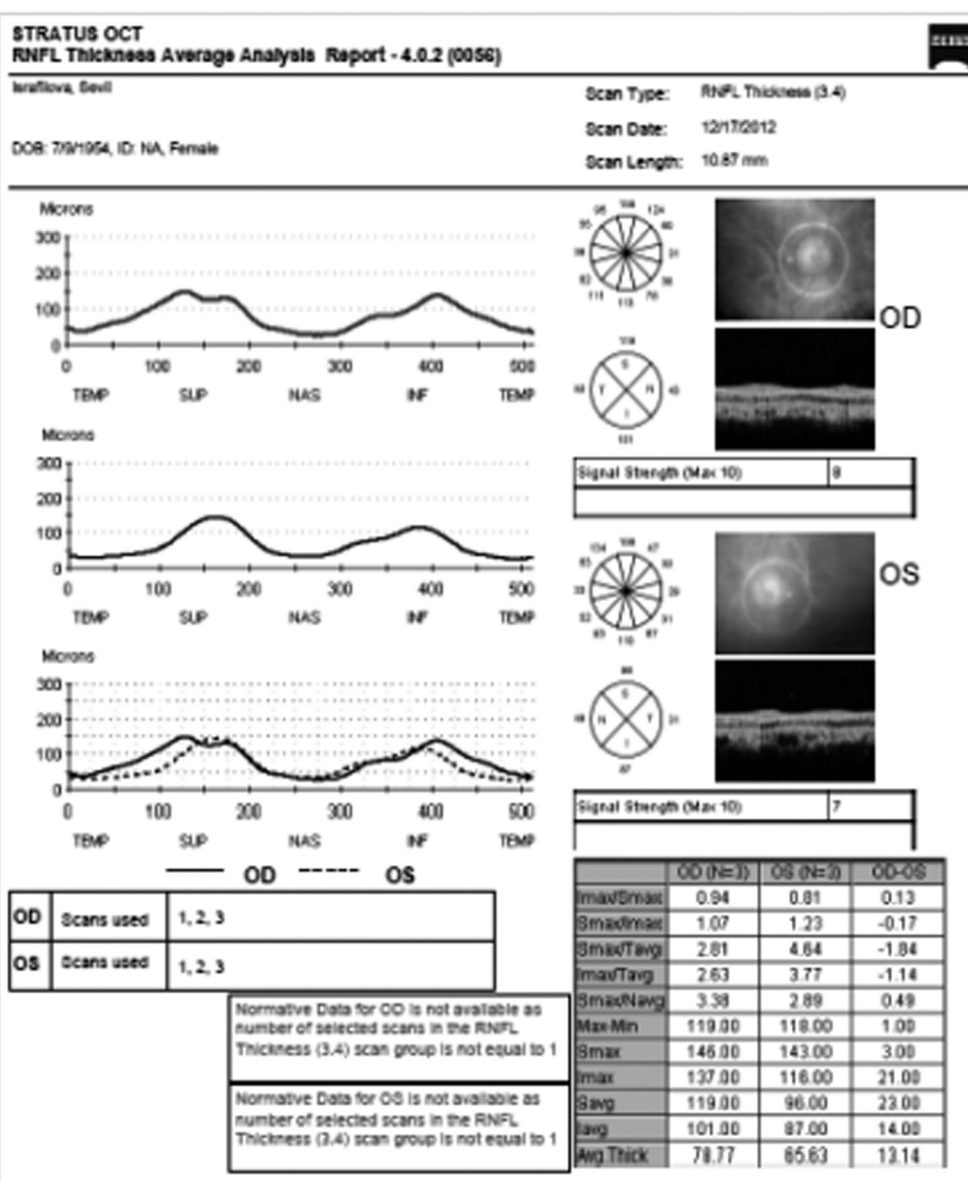

Рис.3. Протокол толщины слоя нервных волокон сетчатки томографии (ОСТ): на обоих глазах было отмечено нарушение правила " ISNT", резкое сокращение толщины слоя нервных волокон сетчатки, причем отмечалось нехарактерное для глаукомы истончение в носовой части, что вкупе с изменениями в полях зрения поставило под сомнение диагноз "нормотензивной глаукомы" (рис.3).

В качестве дополнительного обследования (18.12.12) была выполнена экстракраниальная и транскраниальная ультразвуковая допплерография, которая показала обеднение кровообеспечения головного мозга сосудами круга Виллизия и снижение с левой стороны показателей оттока во внутричерепном сегменте внутренней сонной артерии и в а. ophthalmica. С целью углубленного диагностического поиска пациентке (21.12.12) была произведена магнитно-резонансная томография (МРТ) головного мозга. В ходе исследования была обнаружена макроаденома гипофиза с эндо-, супраи параселлярным (левосторонним) ростом, размерами 32-29-26 мм, сдавливающая область перекреста зрительных нервов и вызвавшая компрессию зрительного нерва левого глаза. Также было отмечено наличие в лобно-теменной области подкожных атером. Изучение гормонального статуса не выявило активного характера аденомы.

Учитывая размеры внутричерепного 


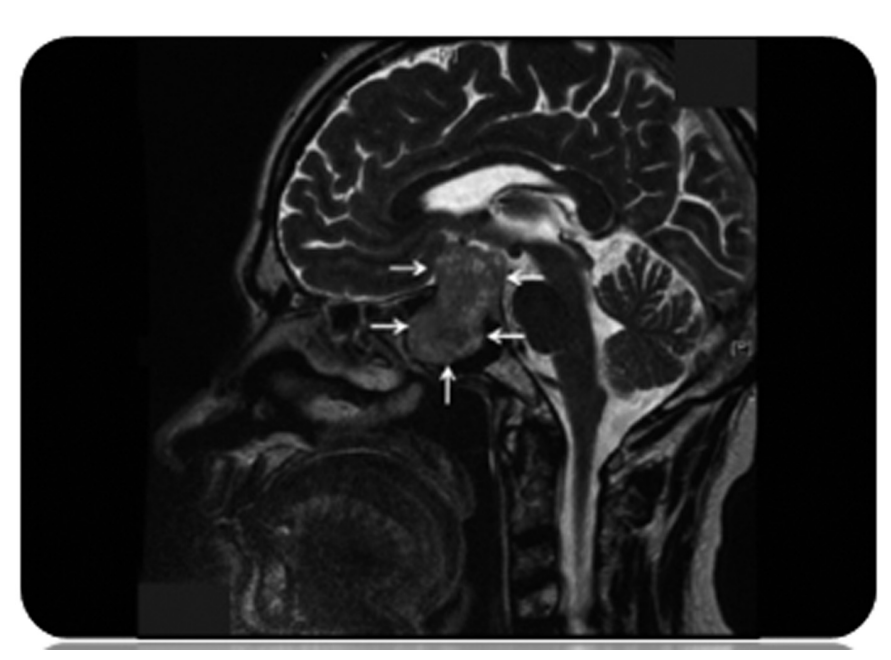

Рис. 4. МРТ головного мозга

образования, прогрессивное развитие зрительных нарушений пациентке была рекомендована и осуществлена транскраниально операция по удалению аденомы гипофиза. На повторном обследовании, спустя 2 месяца, после операции 02.04.13 vis $\mathrm{OD}=0,015 /$ vis $\mathrm{OS}=$ движение руки (экс.) уровень офтальтонуса OD/OS=18/20 мм.рт.ст.(без капель). При обследовании глазного дна была обнаружена полная атрофия ДЗН на обоих глазах, соотношение Э/Д обоих глаз без изменений, калибр сосудов 1:3.

Результаты. Представленный клинический случай и интерпретация нижеизложенного позволяет расставить акценты в дифференциальной диагностике аденомы гипофиза и глаукомы $[4,5,6,7,8,9,14,15,16]$.

- Несмотря на наличие частичной атрофии зрительного нерва левого глаза и смещение сосудистого пучка в носовую сторону, а также некоторую разницу в Э/Д обоих глаз отмечалось побледнение нейроретинального

\section{ЛИТЕРАТУРА}

1. Pillunat L.E., Stodtmeister R., Wilmanns I. Pressure compliance of the optic nerve head in low tension glaucoma//British Journal of Ophthalmology, 1987. Vol 71. P. 181-187

2. Tariq Farooq Babar, Muhammad Tariq Khan and all. Normal tension glaucoma // Pak J Ophthalmol 2006, Vol. 22 No.2.P.60-67

3. Jordan Rixen, Young H. Kwon. Normal Tension Glaucoma: 50 year-old female with progressive visual field loss, glaucomatous disc change and normal IOP // EyeRounds.org. August 14, 2008

4. Schmidt D. Differential diagnosis of bitemporal paracentral visual fields defects in "low-tension glaucoma" and tumors of the pituitary gland // Klin Monbl Augenheilkd 1975. Vol. 166. No 4. P.483-488. ободка (rim), что является специфичным только для не- глаукоматозных оптических нейропатий .

- Наличие битемпоральной гемианопсии, не является характерным для глаукомы. В данном случае также имело место сужение носовых половин поля зрения, что указывает на частичное поражение неперекрещенных волокон в хиазме.

- Относительный афферентный зрачковый дефект (зрачок Маркуса-Гунна) возникает только в случаях асимметричного поражения зрительного пути (сетчатка, зрительный нерв, хиазма, зрительный тракт).

- Именно гормонально неактивный характер внутричерепного образования (иначе бы на первый план выступила эндокринная симптоматика еще на ранней стадии микроаденомы) и позволили проявить себя зрительными нарушениями лишь после того, как опухоль, увеличившись в размерах до макроаденомы получила эндо-, супра- и параселлярное распространение и сдавила хиазму и зрительный нерв.

- Важным вспомогательным методом исследования стал МРТ, т.к. благодаря именно этому методу удалось уточнить и верифицировать диагноз.

Выводы. На примере данного клинического случая хотелось бы подчеркнуть важность своевременного и сочетанного ведения специалистами узкого профиля (офтальмологами, невропатологами, эндокринологами и нейрохирургами) пациентов такого рода для ранней и точной диагностики, что может предотвратить высокий процент инвалидизации.

5. Stefaniu I, Nita N, Zemba M. and all. The nonfunctional pituitary adenoma, The optic chiasm syndrome // Oftalmologia 2000. Vol.50. No 1. P. 79-83.

6. Suzannah R. Drummond, Clifford Weir. Chiasmal compression misdiagnosed as normal-tension glaucoma: can we avoid the pitfalls? //International Ophthalmology 2010. Vol. 30. No 2. P. 215-219

7. Richard Bennett G. Diagnosing a deceptive presentation. A patient with a history of surgery for a brain tumor presents with signs of glaucoma that defy classification // Continuing Education. Issue: April 2009

8. Куроедов А.В., Цалкина Е.Б., Журавлева С.И и др. Клинический случай обнаружения гормонально неактивной аденомы гипофиза в ходе обследования пациента 
с подозрением на глаукому// KOFT, Современные методы исследования в офтальмологии. 2006 . № 2. стр. 84-87 9. Нестеров А.П., Алябьева Ж.Ю. Нормотензивная глаукома: современный взгляд на патогенез, диагностику, клинику и лечение. Часть I.// Глаукома .2005.№ 3.стр.66-74 10. Raed Behbehani. Clinical approach to optic neuropathies // Clin. Ophthalmol. 2007. Vol. 1 №3.P. 233-246 11. Choudhari NS, Neog A, Fudnawala V, George R. Cupped disc with normal intraocular pressure: The long road to avoid misdiagnosis // Indian J Ophthalmol. 2011.Vol.59.P.491-497

12. American Cancer Society Review: 1/11/2013 online

13. Черебилло В.Ю. Транссфеноидальная эндоскопи- ческая хирургия в комплексном лечении аденом гипофиза // Международный Эндокринологический Журнал. 2008. т.5 .№17

14. Flora Levin. When Glaucomatous Damage Isn't Glaucoma. Many conditions besides glaucoma can produce the appearance of the disease // Review of ophthalmology. 5/5/2013

15. James C. Tsai. How to Evaluate the Suspicious Optic Disc. Though intraocular pressure is still a valuable measurement in a glaucoma exam, the optic nerve's appearance can be just as vital// Review of ophthalmology. 6/15/2005

16. Астахов Ю.С., Ангелопуло Г.В., Джалиашвили О.А. Глазные болезни: Для врачей общей практики: Справочное пособие. - СПб.: СпецЛит, 2001.- с. 240

\title{
XÜLASə
}

\section{NORMOTENZIV QLAUKOMA VӘ YA HIPPOFIZIN ADENOMASI?}

\author{
M.E.Ofəndiyeva \\ Akad. Zərifə Oliyeva ad. Milli Oftalmoloji Mərkəz, Bakı
}

Məqsəd: hipofizin mikroadenoması və normotenziv qlaukoma zamanı yaranan görmə patologiyasının diferensial diaqnostikasi.

Mateiallar və metodlar. Sol gözündə görmə sahəsinin düşməsi və görmə itiliyinin enməsindən şikayət edən 59 yaşlı pasient nəzarət altına alınmışdır. Standart oftalmolojı müayinələrə əlavə olaraq optik koqerent tomoqrafiya (stratus OCT 3000), ekstrakranial və transkranial ultrasəs dopleroqrafiya və baş beynin maqnit-rezonans tomoqrafiyası da aparılmışdır.

Yekun. Sol gözündə görmə sinirinin atrofiyası müəyyən edilmişdir.

Bu da 32-29-26 mm ölçülü endo-, supra- və parasellyar (sol tərəfli) böyüyən hormonal qeyri-aktiv hipofizin makroadenoma səbəbdən yaranıb.

Nəticələr. Bu kliniki hadisəyə əsaslanaraq, nəzərə çatdırmaq istərdim ki, əlilliyin faizinin yüksəlməsinin qarşısını almaq və belə pasientlərdə erkən və düzgün diaqnoz qoymaq məqsədi ilə bir neçə dar ixtisas mütəxəssislərinin (oftalmoloqlar, nevroloqlar, endokrinoloqlar, neyrocərrahlar) vaxtında və birgə uyğun şəkildə işləmələri çox vacibdir.

Açar sözlər: normotenziv qlaukoma, hipofizin makroadenoması.

\section{SUMMARY}

\section{NORMOTENSIVE GLAUCOMA OR PITUITARY ADENOMA?}

\author{
Efendiyeva M.E \\ National Centre of Ophthalmology named after acad. Zarifa Aliyeva
}

Purpose: differential diagnostics of visual abnormalities in adenoma of hypophysis and normotensive glaucoma.

Material and methods: Under observation the patient was I., 59 years with complaints of decreased visual acuity and visual field loss in the left eye. In addition to the standard ophthalmologic examinations were performed: optical coherence tomography (Stratus OCT 3000), extracranial and transcranial doppler ultrasound and magnetic resonance tomography (MRT) of cerebrum.

Results: Was found partial atrophy of the optic nerve of the left eye, which was the result of hormonally inactive pituitary macroadenomas sizes 32-29-26 mm with endo-, supra-and parasellyarnym (left-hand) growth. The presented case report allowed to place some accents in the differential diagnosis of pituitary adenoma and normotensive glaucoma Conclusion: On the example of this case report we would like to emphasize the importance of timely and combined management specialists narrow profile (ophthalmologists, neurologists, neurosurgeons and endocrinologists) of the patients of this kind for an early and accurate diagnosis, which can prevent a high percentage of disability.

Key words: normotensive glaucoma, pituitary macroadenoma. 